\title{
Psychogenic Hemiplegia after Cervical Spine Discectomies and Fusion
}

\author{
Siavash S Haghighi* \\ Clinical Neuro-diagnostic Department, Sharp Memorial Hospital, California, USA
}

*Corresponding author: Siavash S Haghighi, Clinical Neuro-diagnostic Department, Orthopedic/Neurosurgery Service, Sharp Memorial Hospital, San Diego, California, USA, Tel: 858-939-3459, Fax: (858)-939-3566

\begin{abstract}
Study design: A case report of psychogenic hemiplegia following a two level anterior cervical discectomies and fusion was contradicted by a thorough electrophysiological findings.

Objective: To report an unusual case of post-operative hemiplegia in spite of normal electrophysiological and noncompromising radiographic findings.

Summary of background data: Conversion disorder with sensory or motor system symptoms is a subtype which includes symptoms such as impaired motor balance, paraplegia, muscle weakness, and urinary retention.

Methods and results: The SSEPs and TCMEPs were recorded intraoperatively in this patient. The SSEPs revealed well-developed peaks for all extremities. TcMEPs was only obtainable from the right intrinsic foot muscles.

Conclusions: In spite of contradictory clinical findings and normal neurophysiologic tests, and normal neuroradiology findings are positive criteria for paraplegia/hemiplegia with psychogenic etiology.
\end{abstract}

\section{Introduction}

Conversion disorder is an unconscious psychological response to a stressful situation [1,2]. The essential feature of conversion disorder is the presence of symptoms or deficits affecting voluntary sensory or motor activity. These symptoms are not intentionally produced [3]. Motor deficits include impaired balance, paralysis, limb weakness, and urinary retention. According to the DMS-5 criteria, following features are identified for psychogenic paralysis etiology: (1) Contradictory clinical findings such as normal muscle tone, normal deep reflex, or pyramidal signs; (2) Paradoxical behavior; (3)
Normal electrophysiological features; and (4) Normal radiographic findings [2,4]. Neurodiagnostic techniques measure brain and spinal cord dysfunction that might otherwise be missed with CT or MRI. They complement imaging techniques by providing a noninvasive measure of the existing physiology [5-7]. At times, physicians are faced with situation in which a patient's symptoms are not completely compatible with the description of the original accident or the physical examination [8-13]. In such cases, electrophysiological examination including modalities such as somatosensory evoked potentials (SSEPs) or transcranial motor evoked potentials (TcMEPs) are helpful in confirming the psychogenic nature of the neurologic deficit $[6,7,14]$.

The present report describes the utility of SSEPs and TCMEPs to rule out an anatomical or electrophysiological cause for paralysis in a patient with Conversion Disorder.

\section{Case Report}

This morbidly obese 47-year-old African-American female was presented to the emergency department with chest pain for about a week. Patient also had increasing difficulty walking and was using a walker. Chest pain was none radiating and patient complained about shortness of breath and dizziness. The patient had a cardiac catheterization which showed normal coronaries.

She also complained about lower back pain severe in intensity and radiating in both of her lower extremities. She reported numbness and tingling in all 4 extremities. She reported a left lower extremity weakness for years

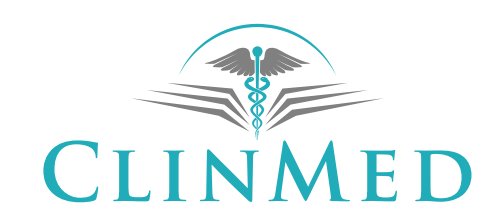

INTERNATIONAL LIBRARY
Citation: Haghighi SS (2019) Psychogenic Hemiplegia after Cervical Spine Discectomies and Fusion. Neurosurg Cases Rev 2:016. doi.org/10.23937/2643-4474/1710016

Accepted: June 06, 2019; Published: June 08, 2019

Copyright: (C) 2019 Haghighi SS. This is an open-access article distributed under the terms of the Creative Commons Attribution License, which permits unrestricted use, distribution, and reproduction in any medium, provided the original author and source are credited. 


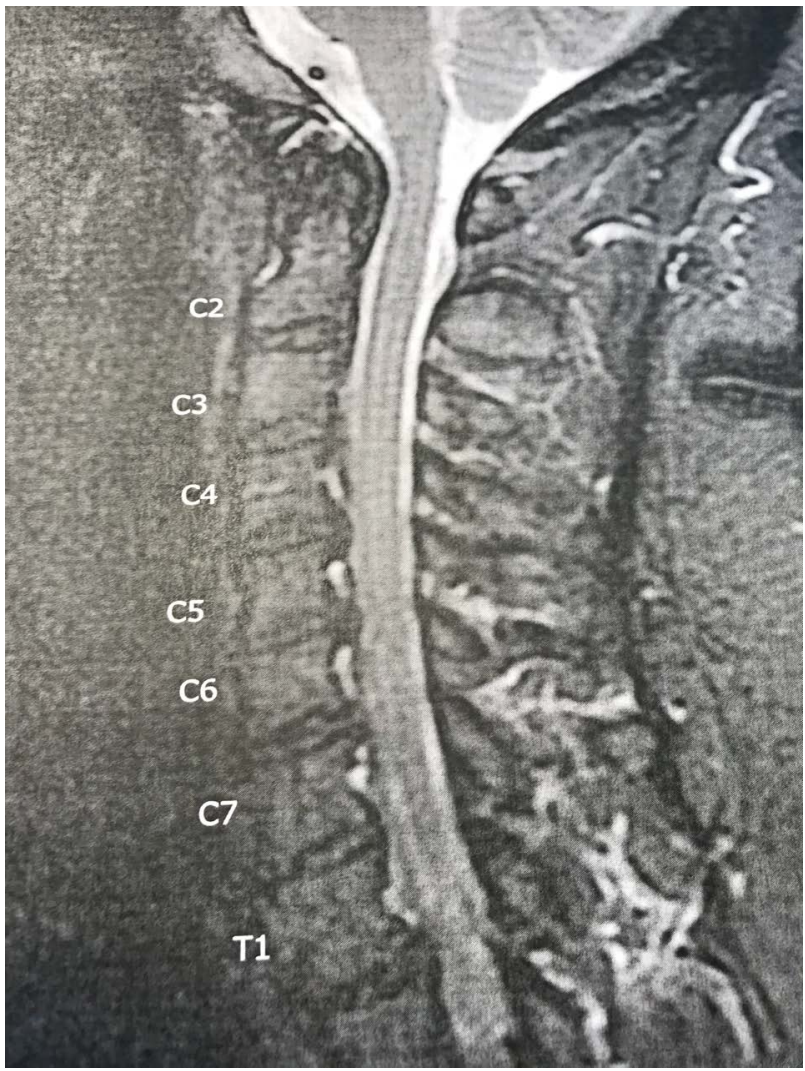

Figure 1: Multilevel degenerative disc disease and spondylosis particularly at C5-6 where there is moderate to severe central canal stenosis. The paraspinous soft tissues were normal in appearance with limited axial images.

and left upper extremity weakness for the last 2 days. She had an MRI of the lumbar spine prior to this admission which demonstrated lumbar spinal stenosis and mild disc prolapse. She also had an MRI of the cervical spine which showed multilevel degenerative disc disease and spondylosis. Particularly at C5-6 where there was a moderate to severe central canal stenosis and a mild neural foramina stenosis with a small prolapse (Figure 1).

Past medical history included, Asthma, diabetes mellitus type 2, morbid obesity, and hypertension. Neurological examination demonstrated strength in left lower extremity $2 / 5$ and unable to lift against gravity and left-sided foot drop. Right lower extremity strength $5 / 5$ and upper extremity strength normal. The patient was then underwent a two level anterior cervical discectomies and fusion without any adverse anesthesia or surgical related events. Under anesthesia the SSEPs and TcMEPs were recorded from the upper/lower extremities during different stages of operation. To evaluate spinal cord function in this case, baseline upper extremity (median nerve; MN) and lower extremity (posterior tibial nerve; PTN) cortical evoked responses were recorded. The alternating electrical pulses were delivered at the wrist region and posterior medial malleolus for the $\mathrm{MN}$ and PTN stimulations. The main cortical peak of N22 msec after the MN stimulation and cortical peak of N45 msec after PTN were noted. The TcMEP was performed by train pulses of 5 at $20 \mathrm{msec}$ duration with corkscrew shaped stimulating leads placed at C3 and C4 cortical locations. The electrical threshold to evoked motor response from distal foot muscles (AH) was around 230 $\mathrm{mA}$. No significant changes in the SSEPs were noted during the procedure. TCMEP was only recordable from the right-sided $\mathrm{AH}$ muscles (Figure 2).

Post-operatively, she was admitted to the ICU. After arriving to the ICU, she complained of increasing right leg weakness and numbness. She also complained of increasing left upper extremity weakness and numbness. An urgent brain MRI was ordered then which demonstrated no acute infarct or ischemic changes in the brain tissue. MRI of the cervical spine revealed expected postoperative changes without any spinal cord signal abnormality. Neurological examination revealed power $2 / 5$ in the right upper extremity, $2 / 5$ in the right lower extremity. $0 / 5$ left lower extremity. 0/5 left upper extremity. The remained in the ICU for 3 days with slow but progressive subjective improvements in sensation and motor function. Patient was transferred to the rehabilitation service in five days after operation. She was also evaluated by the psychiatric service for treatment of depression and conversion disorders. The patient was discharged one week later; she was ambulating normally.

\section{Discussion}

A diagnosis of Conversion Disorder should be made only after a thorough medical investigation has been performed to rule out a neurological or general medical condition $[2,4]$. Conversion symptoms are often inconsistent. A "paralyzed" extremity will be moved inadvertently while dressing, or when attention is as Conversion Disorder. Prominent among them are multiple sclerosis, myasthenia gravis, and idiopathic or substance-induced dystonia. As many as one-third of directed elsewhere. A broad range of neurological conditions may be misdiagnosed individuals with conversion symptoms have a current or prior neurological condition. Individuals with this disorder may show la belle indifference or lack of concern about the nature or implications of their symptoms. No specific laboratory abnormalities are associated with this condition. Psychogenic paralysis after minor trauma presents a difficult medico legal issue. Despite normal films and physical examination, the physician is forced to perform myelography to exclude lesions such as disk herniation, epidural hematoma, or contusion of the spinal cord $[11,15]$. It is the absence of expected findings that suggests and supports the diagnosis of Conversion Disorder. Symptoms typically do not conform to known anatomical pathways and physiological mechanisms. The expected objective signs such as reflex changes following paralysis are rarely present. Conversion paralysis after cervical disc decompression and fusion is exceedingly rare occasion but reported in the literature [15]. The differential diagnosis of conversion disorder requires exclusion of other neurological disease, including strokes, multiple sclerosis, and epilepsy $[1,2]$. 

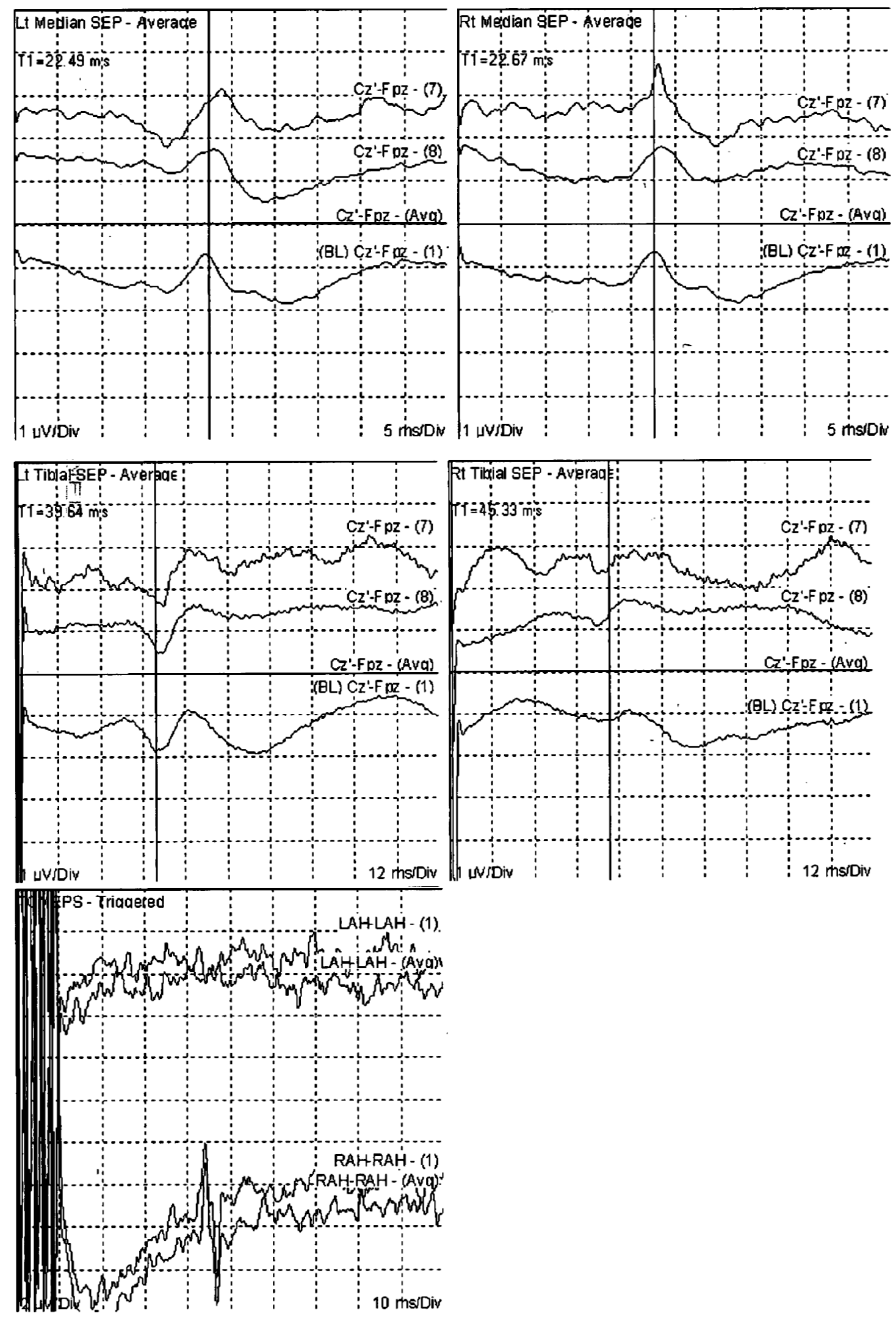

Figure 2: The somatosensory evoked potentials (SSEPs) were recorded by alternately stimulating each posterior tibial nerve (PTN) at the ankle region behind the medial malleolus or the median nerve $(\mathrm{MN})$ at the wrist region. The SSEPs revealed well-developed cortical peaks for all four extremities. Transcranial cortical stimulation (TcMEPs) demonstrated evoked muscle response at the right adductor halluces $(\mathrm{AH})$ muscle on the right side only.

SSEP and MEP recording are simple and non-invasive methods which can objectively evaluate integrity of central and peripheral sensory and motor pathways $[7,6,14]$. The use of SSEPs has expanded to the evalua- 
tion of trauma, stroke, tumors, and metabolic disorders [5]. Morota, et al. reported a case of psychogenic quadriparesis diagnosed by MEP monitoring in a 12-year-old girl who underwent surgery for repair of a recurrent syringomyelia. The SSEP test showed no change in comparison to those obtained at the end of her preceding surgery. MEP was normal for the lower extremities, a finding which was inconsistent with a severe upper motor neuron lesion [7]. Our single case report in a psychogenic paraplegia patient demonstrated a complete and well defined SSEPs contradicting her paralysis [6]. This current report also shows the value of electrophysiological recordings to diagnose conversation disorder in our patient.

In Conclusion, normal SSEPs or MEPs in a patient denying sensation of the stimulus offer objective evidence of the psychogenic nature of the para/quadriplegia and are a valuable tool in this clinical setting. The rehabilitation of patients with conversion paraplegia should include physical therapy and reassurance. Psychiatric consultation is important to define underlying psychopathology.

\section{References}

1. Afolabi K, Ali Sameer, Gahtan V, Gorji R, Li F, et al. (2016) Postoperative conversion disorder. Journal of Clinical Anesthesia 30: 21-23.

2. Baker JHE, Silver JR (1987) Hysterical paraplegia. Journal of neurology, Neurosurgery, and Psychiatry 50: 375-382.

3. American Psychiatric Association (1994) Conversion Disorders. In: Diagnostic and statistical manual of mental disorders. ( $4^{\text {th }}$ edn), American Psychiatric Association, Washington, USA.

4. Ford CV (2000) Somatoform disorders. In: Ebert MH, Current diagnosis and treatment in psychiatry. ( $1^{\text {st }}$ edn), McGraw-Hill, New York, 366-377.
5. Chiappa KH, Ropper AH (1982) Evoked potentials in clinical medicine (first of two parts). N Engl J Med 306: 1140-1150.

6. Haghighi SS, Meyer S (2001) Psychogenic paraplegia in a patient with normal electrophysiological findings. Spinal Cord 39: 664-667.

7. Morota N, Deletis V, Kiprovski K, Epstein F, Abbott R, et al. (1994) The use of motor evoked potentials in the diagnosis of psychogenic quadriparesis. A case study. Pediatric Neurosurg 20: 203-206.

8. Shell MG, Mitchell HL, Loes MW, Belan AP (1997) Conversion disorder presenting in a patient with an implantable morphine pump and an epidural abscess resulting in paraplegia. Arch Phys Med Rehabil 78: 226-229.

9. Berhane L, Kurman R, Smith S (1998) Lower extremity paralysis after operative laparoscopy from conversion disorder. A case report. J Reprod Med 43: 831-835.

10. Crimlisk HL, Bhatia K, Cope H, David A, Marsden CD, et al. (1998) Salter revisited: 6 year follow up study of patients with medically unexplained motor symptoms. BMJ 316 : 582-586.

11. Laraki M, Orliaguet GA, Flandin C, Merkx J, Barrier G (1996) Hysterical paraplegia as a cause of transient paraplegia after epidural anesthesia. Anesth Analg 83: 876-877.

12. Rappaport M, Sweeney D, Rappaport ML, Saal J, Wilmot C (1998) Neuropsychiatric assessment of a spinal cord injury patient with sudden recovery. Arch Phys Med Rehabil 69: 455-457.

13. Solyom C, Solyom L (1990) A treatment program for functional paraplegia/Munchausen syndrome. J Behav Ther Exp Psychiatry 21: 225-230.

14. Kaplan BJ, Friedman WA, Gravenstein D (1985) Somatosensory evoked potentials in hysterical paraplegia. Surg Neurol 23: 502-506.

15. Boudissa M, Castelain JE, Boissiere L, Mariey R, Pointillart V, et al. (2015) Conversion paralysis after cervical spine arthroplasty: A case report and literature review. Orthopedics \& Traumatology Surgery \& Research 101: 637-641. 